\title{
Minimally invasive explantation of implantable left ventricular assist devices
}

Patrick Tansley, BSc(Hons), FRCS(Eng), and Magdi Yacoub, FRS, Harefield, Middlesex, United Kingdom

I nterest in the management of dilated cardiomyopathy has recently been enhanced by the development of bridge to recovery techniques that use mechanical circulatory support. ${ }^{1-5}$ A previously reported program of this type, combining mechanical with pharmacologic support involving induction of reverse remodeling followed by physiologic hypertrophy, has been developed at Harefield Hospital. ${ }^{6}$ After recovery, explantation of left ventricular assist devices has traditionally comprised median sternotomy and laparotomy 7,8 with the associated extensive dissection of adhesions that can increase morbidity and mortality. To avoid these complications, we have developed and evaluated a new minimally invasive approach.

\section{Method}

The apex of the heart is exposed through an anterolateral minithoracotomy (Figure 1). Two other separate small incisions are made: an epigastric incision to expose the device and a limited anterior thoracotomy through the second intercostal space to expose the aortic anastomosis of the outflow cannula (Figure 2). Femorofemoral cardiopulmonary bypass is established, and the heart is fibrillated electrically to allow explantation of the device inflow cannula. After deairing, which is easily accomplished by rotation of the operating table to the right and partial constriction of the venous return cannula, the apex is repaired by direct suture (Figure 3). The heart is then defibrillated electrically with external or small internal paddles. Cardiopulmonary bypass is then discontinued, with strict monitoring of pulmonary arterial pressure by means of a thermodilution catheter (Swan-Ganz; Baxter Healthcare Corp, Edwards Division, Santa Ana, Calif). The outflow graft is divided as close as possible to the aortic anastomosis. The residual graft stump, which measures 2 to $3 \mathrm{~mm}$ in length, is oversewn (Figure 4 ), and the device is then explanted, along with the intrathoracic part of the Dacron polyester fabric graft, by traction applied from below (Figure 5).

\section{Results}

During the past 18 months we have used this technique to explant devices from 12 consecutive patients and have studied these cases

From Harefield Hospital, Middlesex, United Kingdom.

Received for publication Jan 4, 2002; accepted for publication Jan 5, 2002.

Address for reprints: Professor Sir Magdi Yacoub, Heart Science Centre, Harefield Hospital, Middlesex, UB9 6JH, United Kingdom (E-mail: jacqui.scott@rbh.nthames.nhs.uk; m.yacoub@ic.ac.uk).

J Thorac Cardiovasc Surg 2002;124:189-91

Copyright (C) 2002 by The American Association for Thoracic Surgery

$0022-5223 / 2002 \$ 35.00+0 \quad \mathbf{1 2 / 5 4 / 1 2 2 7 8 6}$

doi: $10.1067 / \mathrm{mtc} .2002 .122786$

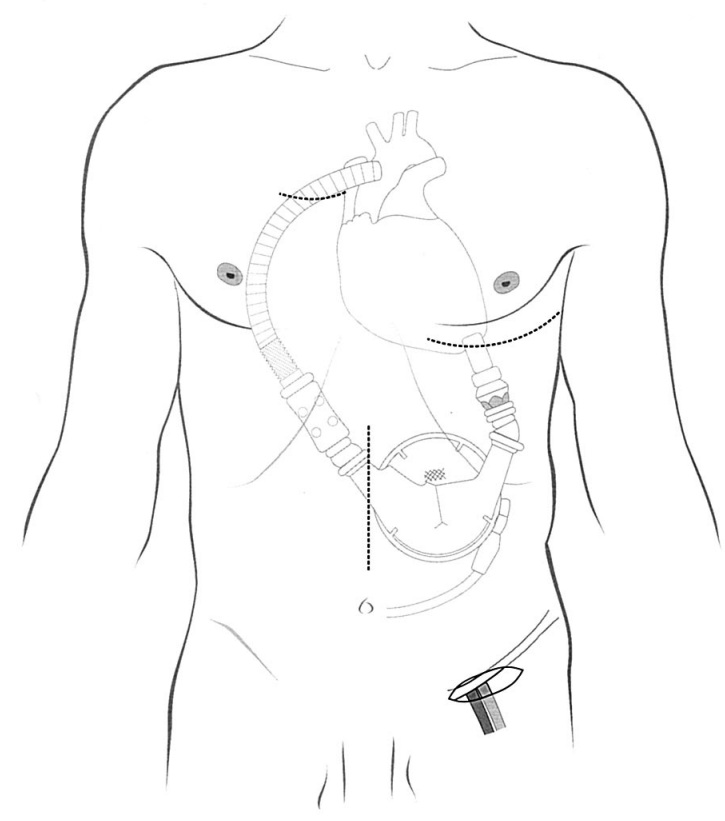

Figure 1. Diagram showing sites of minimally invasive incisions and arteriovenous cannulation in groin.

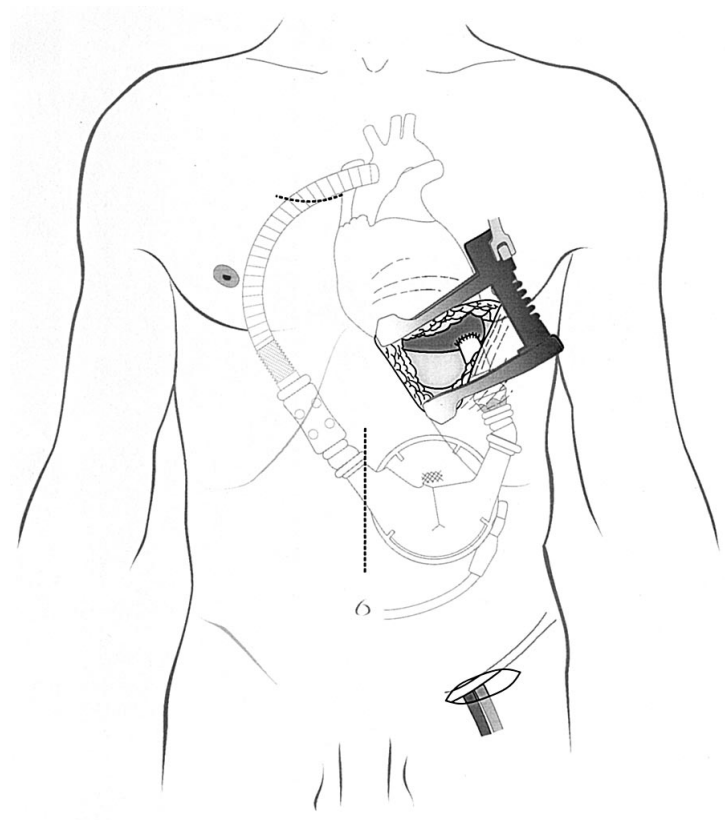

Figure 2. Diagram showing dissection of inflow cannula through minimally invasive anterior thoracotomy. Cannula is then extracted during short period of cardiopulmonary bypass. 


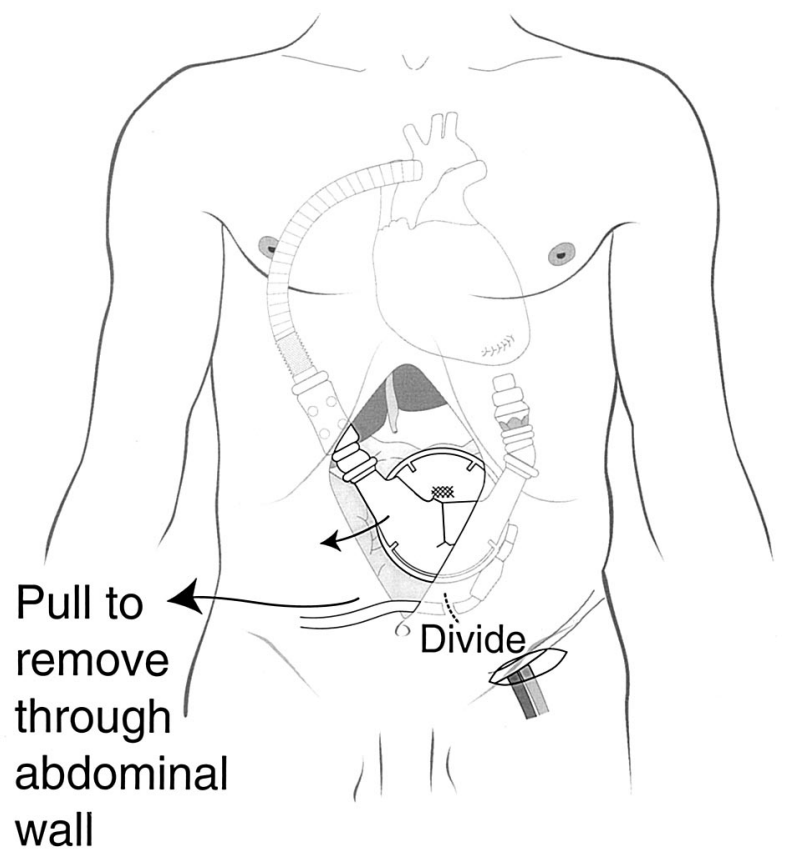

Figure 3. Drive line is divided close to device and is removed by traction from outside.

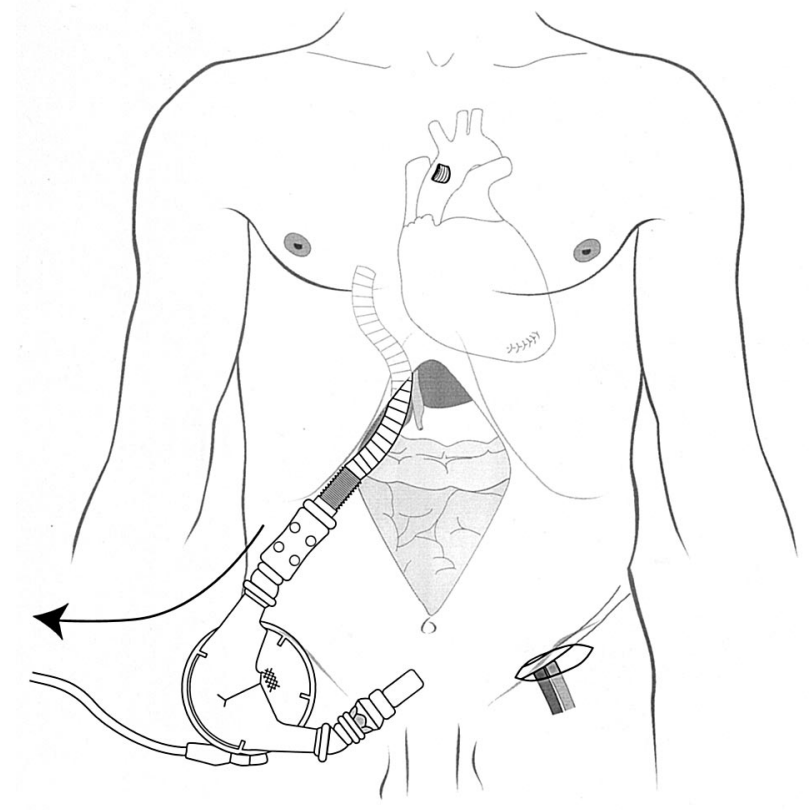

Figure 5. Finally, device is completely removed by traction on outflow graft from below.
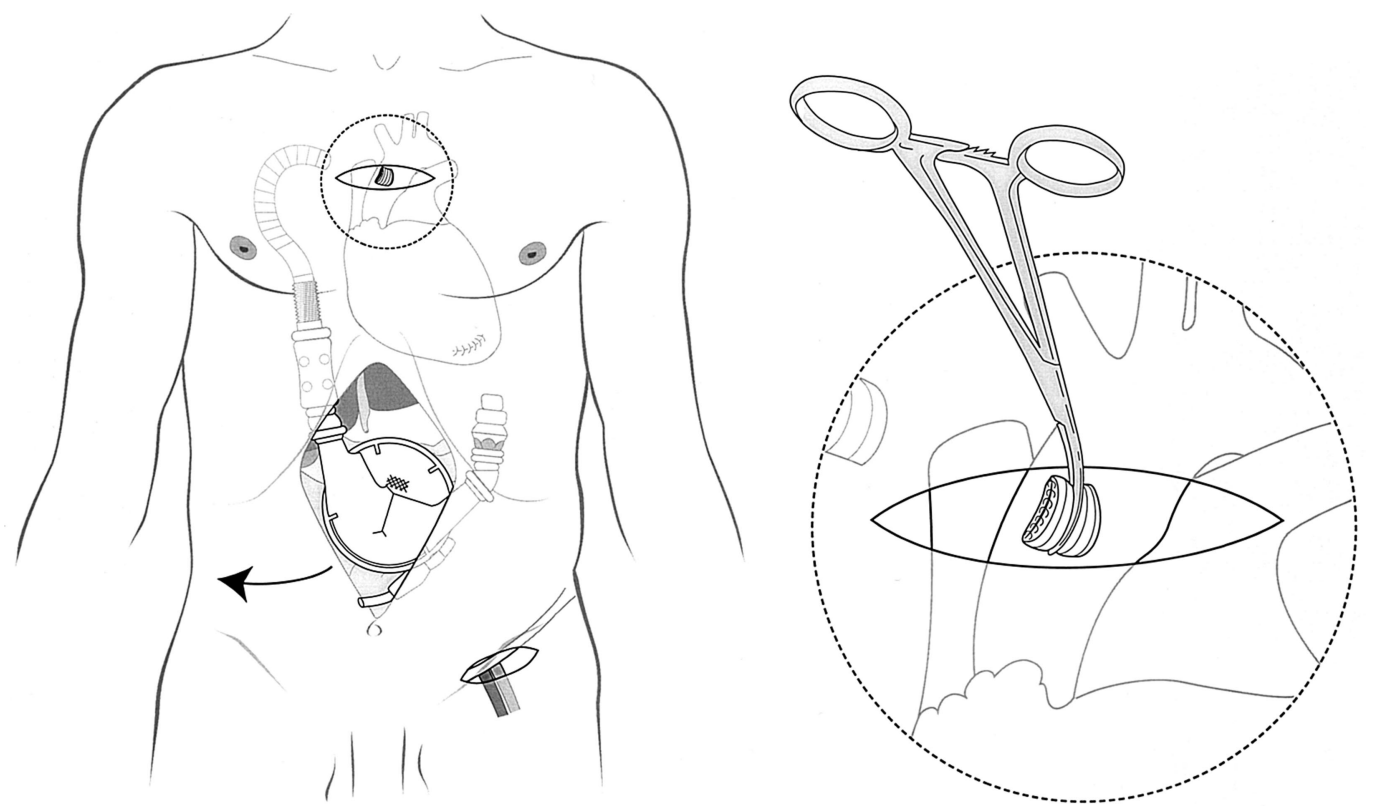

Figure 4. Body of device is explanted after dissection around diaphragmatic passage of inflow cannula. Outflow cannula is exposed through anterior thoracotomy, then clamped and divided as close as possible to the ascending aorta. Stump is oversewn. An inset to Figure 4 is shown on the right. 
on a prospective basis. All patients were supported by Thoratec HeartMate devices (11 HeartMate I and 1 HeartMate II; Thoratec Laboratories Corporation, Pleasanton, Calif, formerly Thermo Cardiosystems Inc). One patient died early of unrelated causes, and the 11 remaining patients all made a smooth recovery. Mean $( \pm \mathrm{SD})$ intensive treatment unit stay was $6.2 \pm 3.3$ days, with no cases of cerebral dysfunction, thromboembolism, or organ failure. Serious infection was not encountered. One patient had a superficial epigastric wound infection, which was treated conservatively.

\section{Conclusion}

We have evolved and used a simple, minimally invasive approach that appears to be safe, with a low complication rate. Because of increasing interest in bridge to recovery programs, we anticipate that this technique may become increasingly useful in reducing morbidity and mortality during the explantation of these devices.

\section{References}

1. Hetzer R, Muller JH, Weng Y, Meyer R, Dandel M. Bridging-torecovery. Ann Thorac Surg. 2001;71(3 Suppl):S109-13.

2. Kumpati GS, McCarthy PM, Hoercher KJ. Left ventricular assist device bridge to recovery: a review of the current status. Ann Thorac Surg. 2001;71(3 Suppl):S103-8.

3. Frazier $\mathrm{OH}$, Myers TJ. Left ventricular system as a bridge to myocardial recovery. Ann Thorac Surg. 1999;68:734-41.

4. Yacoub MH, Birks EJ, Tansley P, Henein MY, Bowles CT. Bridge to recovery: the Harefield approach. J Congestive Heart Failure Circ Support. 2001;2:27-30.

5. Young JB. Healing the heart with ventricular assist device therapy: mechanisms of cardiac recovery. Ann Thorac Surg. 2001;71(3 Suppl): S210-9.

6. Yacoub MH. A novel strategy to maximize the efficacy of left ventricular assist devices as a bridge to recovery. Eur Heart J. 2001;22:534-40.

7. Oz MC, Levin HR, Rose EA. Technique for removal of left ventricular assist devices. Ann Thorac Surg. 1994;58:257-8.

8. Borovetz HS, Ramasamy N, Zerbe TR, Portner PM. Evaluation of an implantable ventricular assist system for humans with chronic refractory heart failure: device explant protocol. ASAIO J. 1995;41:42-8. 